\title{
Quasi-wetting and morphological phase transitions in confined far-from-equilibrium magnetic thin films
}

\author{
Julián Candia $^{a}$ and Ezequiel V. Albano ${ }^{b}$ \\ ${ }^{a}$ Departamento de Física, UNLP, CC67, 1900 La Plata, Argentina \\ ${ }^{b}$ Instituto de Investigaciones Fisicoquímicas Teóricas y Aplicadas \\ (INIFTA), UNLP, CONICET, Suc.4, CC16, 1900 La Plata, Argentina
}

June 24, 2018

\begin{abstract}
The growth of confined magnetic films with ferromagnetic interactions between nearest-neighbor spins is studied in a stripped $(1+1)$-dimensional rectangular geometry. Magnetic films are grown irreversibly by adding spins at the boundaries of the growing interface. A competing situation with two opposite short range surface magnetic fields of the same magnitude is analyzed. Due to the antisymmetric condition considered, an interface between domains with spins having opposite orientations develops along the growing direction. Such interface undergoes a localization-delocalization transition that is identified as a quasi-wetting transition, in qualitative agreement with observations performed under equilibrium conditions. In addition, the film also exhibits a growing interface that undergoes morphological transitions in the growth mode. It is shown that, as a consequence of the nonequilibrium nature of the investigated model, the subtle interplay between finite-size effects, wetting, and interface growth mechanisms leads to more rich and complex physical features than in the equilibrium counterpart. Indeed, a phase diagram that exhibits eight distinct regions is evaluated and discussed. In the thermodynamic limit, the whole ordered phase (which contains the quasi-wetting transition) collapses, while within the disordered phase, standard extrapolation procedures show that only two regions are present in the phase diagram of the infinite system.
\end{abstract}




\section{Introduction}

The preparation and characterization of magnetic nanowires and films is of great interest for the development of advanced microelectronic devices. Therefore, the study of the behavior of magnetic materials in confined geometries, e.g. thin films, has attracted both experimental $[1,2,3]$ and theoretical $[4,5,6,7]$ attention. On the other hand, the investigation of very interesting wetting phenomena has also drawn enormous attention. For instance, surface enrichment or wetting layers have been observed experimentally in a great variety of systems, such as e.g. polymer mixtures $[8,9]$ and adsorption of simple gases on alkali metal surfaces [10]. Indeed, it is recognized that wetting of solid surfaces by a fluid is a phenomenon of primary importance in many fields of practical technological applications (lubrication, efficiency of detergents, oil recovery in porous material, stability of paint coatings, interaction of macromolecules with interfaces, etc. [11]). Furthermore, the study of wetting transitions at interfaces has also attracted considerable theoretical interest $[12,13,14]$, involving, among others, different approaches such as the mean field Ginzburg-Landau method [15, 16], transfer matrix and Pfaffian techniques [17, 18], density matrix renormalization group methods [19], solving the Cahn-Hilliard equation [20], using Molecular Dynamic simulations [21], solving self-consistent field equations [22], and by means of extensive Monte Carlo simulations [4, 23, 24, 25, 26].

However, most of the theoretical work has been carried out within the framework of equilibrium systems. In contrast, the aim of this work is to study the properties of thin magnetic film growth under far-from-equilibrium conditions, using extensive Monte Carlo simulations. In order to simulate thin film growth, our study is carried out in confined (stripped) geometries, which resemble recent experiments where the growth of quasi-one-dimensional strips of $\mathrm{Fe}$ on a $\mathrm{Cu}(111)$ vicinal surface [1] and Fe on a W(110) stepped substratum [3] have been performed. Also, in a related context, the study of the growth of metallic multilayers have shown a rich variety of new physical phenomena. Particularly, the growth of magnetic layers of $\mathrm{Ni}$ and Co separated by a $\mathrm{Cu}$ spacer layer has recently been studied [27]. It should also be remarked that, although the discussion is presented here in terms of a magnetic language, the relevant physical concepts can be extended to other systems such as fluids, polymers, and binary mixtures.

In the present work it is shown that, in far-from-equilibrium systems, the subtle interplay between finite-size effects, wetting, and interface growth mechanisms leads to more rich and complex physical features than in the equilibrium counterpart. In fact, a complex phase diagram, that exhibits a localization-delocalization transition in the interface that runs along the walls and a change of the curvature of the growing interface running perpendicularly to the walls, is evaluated and discussed.

This manuscript is organized as follows: in Section II we give details on the simulation method, Section III is devoted to the presentation and discussion of the 


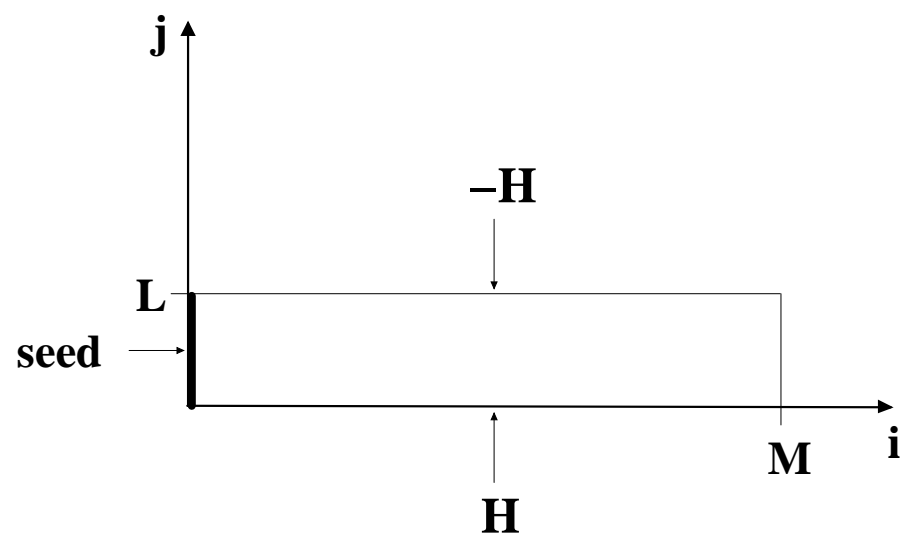

Figure 1: The general set-up for the MEM in a $(1+1)$-dimensional rectangular geometry. The magnetic film grows along the positive longitudinal direction from a seed constituted by $L$ parallel-oriented spins placed at $i=1$, as indicated. Open boundary conditions are assumed along the transverse direction, in which competing surface magnetic fields $H>0\left(H^{\prime}=-H\right)$ acting on the sites placed at $j=1(j=L)$ are considered. Since all deposited spins are frozen, an algorithm that shifts the active growing region towards low $i$ values can be repeatedly applied when the film is close to reaching the limit of the sample $(i=M)$. Hence, finite-size results are independent of $M$, and are thus only governed by the lattice width $L$.

results, while the conclusions are finally stated in Section IV.

\section{The model and the simulation method}

In the classical Eden model [29] on the square lattice, the growth process starts by adding particles to the immediate neighborhood (the perimeter) of a seed particle. Subsequently, particles are stuck at random to perimeter sites. This growth process leads to the formation of compact clusters with a self-affine interface [30, 31, 32, 33]. The growth of a ferromagnetic material can be studied by means of the so called magnetic Eden model (MEM) [34], which considers an additional degree of freedom due to the spin of the growing particles. In the present work the MEM is investigated on the square lattice using a rectangular geometry $L \times M$ (with $M \gg L[28]$ ).

Figure 1 illustrates the general set-up assumed. The location of each site on the lattice is specified through its rectangular coordinates $(i, j),(1 \leq i \leq M, 1 \leq j \leq L)$. The starting seed for the growing cluster is a column of parallel-oriented spins placed at $i=1$ and film growth takes place along the positive longitudinal direction (i.e. $i \geq 2$ ). The boundary conditions are open along the transverse direction, in which competing surface magnetic fields $H>0\left(H^{\prime}=-H\right)$ acting on the sites placed at 
$j=1(j=L)$ are considered. Then, magnetic films are grown by selectively adding spins $\left(S_{i j}= \pm 1\right)$ to perimeter sites, which are defined as the nearest-neighbor $(\mathrm{NN})$ empty sites of the already occupied ones.

Considering a ferromagnetic interaction of strength $J>0$ between NN spins, the energy $E$ of a given configuration of spins is given by

$$
E=-\frac{J}{2}\left(\sum_{\left\langle i j, i^{\prime} j^{\prime}\right\rangle} S_{i j} S_{i^{\prime} j^{\prime}}\right)-H\left(\sum_{\left\langle i, \Sigma_{1}\right\rangle} S_{i 1}-\sum_{\left\langle i, \Sigma_{L}\right\rangle} S_{i L}\right),
$$

where the summation $\left\langle i j, i^{\prime} j^{\prime}\right\rangle$ is taken over occupied NN sites, while $\left\langle i, \Sigma_{1}\right\rangle,\left\langle i, \Sigma_{L}\right\rangle$ denote summations carried over occupied sites on the surfaces $j=1$ and $j=L$, respectively. Throughout this work we set the Boltzmann constant equal to unity and we take the temperature, energy, and magnetic fields measured in units of $J$. The probability for a perimeter site to be occupied by a spin is taken to be proportional to the Boltzmann factor $\exp (-\Delta E / T)$, where $\Delta E$ is the change of energy involved in the addition of the given spin. At each step, the probabilities of adding up and down spins to a given site have to be evaluated for all perimeter sites. After proper normalization of the probabilities, the growing site and the orientation of the spin are determined through standard Monte Carlo techniques. Although both the interaction energy and the Boltzmann probability distribution considered for the MEM are similar to those used for the Ising model with surface magnetic fields [4], it must be stressed that these two models operate under extremely different conditions, namely the MEM describes the irreversible growth of a magnetic material and the Ising model deals with a magnet under equilibrium. In the MEM, the position and orientation of all deposited spins remain fixed. During the growth process, the system develops a rough growth interface and evolves mainly along the longitudinal direction. Some lattice sites can remain empty even well within the system's bulk, but, since at each growth step all perimeter sites are candidates for becoming occupied, these holes are gradually filled. Hence, far behind the active growth interface, the system is compact and frozen. When the growing cluster interface is close to reaching the limit of the sample $(i=M)$, the relevant properties of the irreversibly frozen cluster's bulk (in the region where the growing process has definitively stopped) are computed, the useless frozen bulk is thereafter erased, and finally the growing interface is shifted towards the lowest possible longitudinal coordinate. Hence, repeatedly applying this procedure, the growth process is not limited by the lattice length $M$, and so the lattice width $L$ is the only relevant parameter concerning the finite-size nature of the sample [28]. In the present work clusters having up to $10^{8}$ spins have typically been grown. 


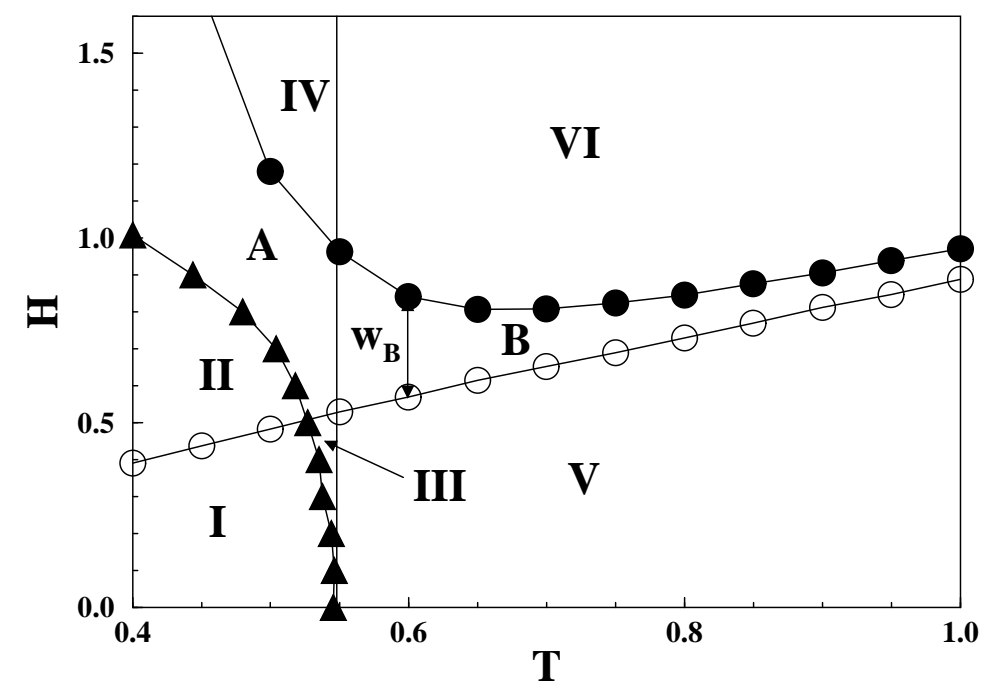

Figure 2: $H-T$ phase diagram corresponding to a lattice of size $L=32$. The vertical straight line at $T_{c}(L)=0.55$ corresponds to the $L$-dependent critical temperature, which separates the low-temperature ordered phase from the high-temperature disordered phase. Open (filled) circles refer to the transition between non-defined and convex (concave) growth regimes, and triangles stand for the Ising-like localizationdelocalization transition curve. Eight different regions are distinguished, as indicated in the figure. Also $w_{B}$, the isothermal width of Region $B$, is marked for $T=0.6$. More details in the text.

\section{Results and discussion}

Magnetic Eden films that grow in a confined geometry with competing surface fields exhibit a very rich phase diagram, which is composed of eight regions (as shown in figure 2). These regions are delimited by several distinct, well defined transition curves. As will be shown below, the bulk order-disorder (finite-size) critical point $T_{c}(L)$, the Ising-like quasi-wetting transition curve $T_{w}(L, H)$, and two morphological transitions associated to the curvature of the growing interface (namely, from convex to non-defined to concave), can be quantitatively located. Moreover, in order to gain some insight into the physics involved in this complex phase diagram, some typical snapshot configurations characteristic of the various different growth regimes are obtained (see figure 6) and discussed.

As well known from finite-size scaling theory, there is some degree of arbitrariness in locating the $L$-dependent critical temperature $T_{c}(L)$ of a finite system. However, the critical point $T_{c}$ of the infinite system, obtained by extrapolating $T_{c}(L)$ to the $L \rightarrow \infty$ limit, is unique and independent of any particular choice for the finitesize critical point. In particular, the ( $L$-dependent) bulk order-disorder critical 
temperature can be identified with the peak of the susceptibility at zero surface field. For $L=32$, the critical point so defined is $T_{c}(L=32)=0.55$, and is shown in figure 2 by a vertical straight line. So, the left (right) hand side part of the phase diagram corresponds to the ordered (disordered) growth regime that involves Regions $I, I I, I I I, I V$, and $A$ (Regions $V, V I$, and $B$ ).

Previous studies [35] have demonstrated that the MEM in a stripped $(1+$ 1)-dimensional geometry is not critical (i.e. it only exhibits an ordered phase at $T=0$ in the thermodynamic limit). Hence, as we consider larger and larger lattices, the finite-size critical points $T_{c}(L)$ turn smaller and smaller, and vanish indeed in the thermodynamic limit. This implies that the eight regions coexisting in the $H-T$ phase diagram are a finite-size effect only relevant for the growth of magnetic films in confined geometries. The ordered phase corresponding to the $T<T_{c}(L)$ region becomes steadily narrower the larger the lattice width $L$, and in the $L \rightarrow \infty$ limit only the disordered phase corresponding to the $T>T_{c}(L)$ region survives. Furthermore, as will be shown below, also Region $B$ shrinks and collapses in this limit, so that only Regions $V$ and $V I$ are present in the phase diagram of the infinite system.

As in previous investigations [35], let us define the mean transverse magnetization $m(i, L, T, H)$ as

$$
m(i, L, T, H)=\frac{1}{L} \sum_{j=1}^{L} S_{i j} .
$$

Also the susceptibility $\chi$ can be defined, as usual, in terms of the magnetization fluctuations. Then, using a standard procedure [4], the localization-delocalization transition curve corresponding to the up-down interface running along the walls can be computed considering that on the $H-T$ plane, a point with coordinates $\left(H_{w}, T_{w}\right)$ on this curve maximizes $\chi(H, T)$. So, the size-dependent localization-delocalization transition curve is obtained, as shown in figure 2 (curve with triangles). As in the case of the Ising model, this quasi-wetting transition refers to a transition between a nonwet state that corresponds to a localized interface bound to one of the confinement walls, and a wet state associated to a delocalized domain interface centered between roughly equal domains of up and down spins $[4,16]$. In fact, it is observed a finite jump in the wetting layer thickness that takes place as a result of the finite size of the system. As the lattice size is increased, the magnitude of the jump grows. However, it should be remarked again that the occurrence of this phenomenon at finite temperature is essentially due to the small size of the thin film, and it becomes irrelevant in the thermodynamic limit. Since confined $(1+1)-$ dimensional magnetic Eden films are noncritical [35], the whole ordered phase corresponding to $T<T_{c}(L)$ (involving Regions $I, I I, I I I, I V$, and $A$ ) that also contains the quasi-wetting curve, collapses in the $L \rightarrow \infty$ limit. Thus, standard procedures carried out in the investigation of equilibrium wetting phenomena concerning the finite-size scaling behavior within the ordered phase $[4,15,16,17,18,19,20,21,22,23,24,25,26]$ are simply 

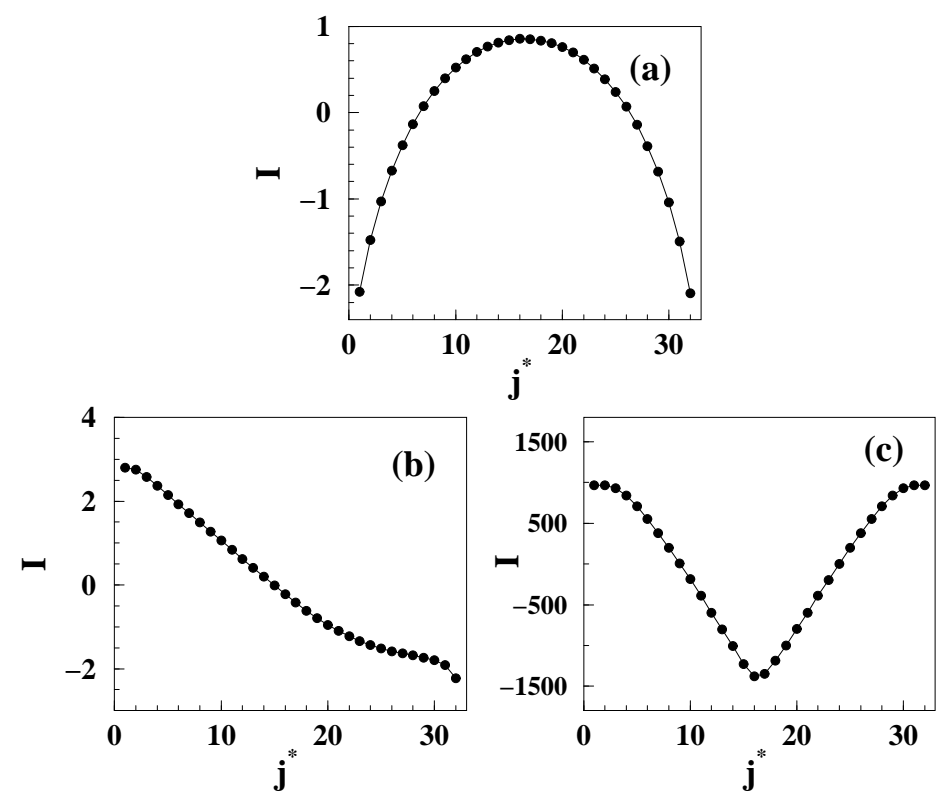

Figure 3: Plots of the averaged interface profile $I$ vs $j^{*}$ for $T=0.6, L=32$ and different values of the surface magnetic field $H$ : (a) $H=0$, (b) $H=0.6$, and (c) $H=4$. The side $j^{*}=1\left(j^{*}=L\right)$ is the one associated with the dominant (nondominant) spin domain. Increasing the surface fields, the curvature of the growing interface changes: convex (a) $\rightarrow$ non-defined (b) $\rightarrow$ concave (c). This qualitative behavior has been observed for all temperatures and lattice sizes within the range of interest of this work.

meaningless in the case of the model investigated here.

Since the MEM is a nonequilibrium kinetic growth model, it also allows the identification of another kind of phase transition, namely a morphological transition associated with the curvature of the growing interface of the system. To avoid confusion, we remark that the term interface is used here for the transverse interface between occupied and empty lattice sites, while it was used above for the longitudinal interface between up and down spin domains.

Figure 3 shows the shape of the mean growing interface $I$ obtained for different values of the surface magnetic field $H$, for fixed temperature $(T=0.6)$ and lattice size $(L=32)$. Notice that the transverse coordinate has been conveniently redefined, so that $j^{*}=1\left(j^{*}=L\right)$ corresponds to the side of dominant (non-dominant) spin domain, while $j=1(j=L)$ is the side of positive (negative) magnetic field. ¿From the figure it follows that three qualitatively distinct growth regimes can clearly be distinguished. Indeed, it is observed that, while for small fields the system grows with convex curvature, increasing the fields the growth process enters into a regime of non-defined curvature, since the dominant spin domain partially wets 


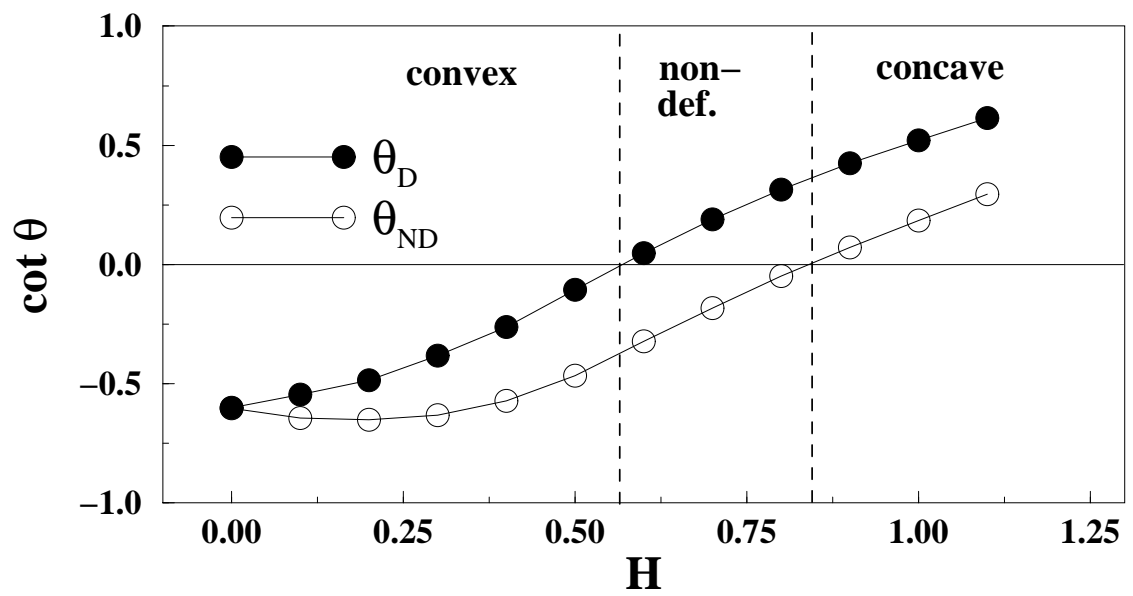

Figure 4: Plots of $\cot (\theta)$ vs $H$ for $T=0.6$ and $L=32 . \theta_{D}\left(\theta_{N D}\right)$ is the contact angle corresponding to the dominant (non-dominant) spin cluster, and is represented by open (filled) circles. The vertical dashed lines mark the fields that separate a given growth regime from another one, as indicated. A reference line corresponding to $\cot (\theta)=0$ has also been included.

the confinement wall, while the non-dominant domain does not. But then, further increasing the fields, a point is reached where the non-dominant spin domain also (partially) wets the wall and the growing interface turns concave. This qualitative behavior has been observed for all temperatures and lattice sizes within the range of interest of this work.

To explore this phenomenon quantitatively, the behavior of the contact angles between the growth interface and the confinement walls (as functions of temperature and magnetic field) have to be investigated. Clearly, two different contact angles should be defined in order to locate this transition, namely $\theta_{D}$ for the angle corresponding to the dominant spin cluster, and $\theta_{N D}$ for the one that corresponds to the non-dominant spin cluster. Figure 4 shows plots of $\cot (\theta)$ vs $H$ for $T=0.6$ and $L=32$. The vertical dashed lines indicate the fields that separate a given growth regime from another one. One observes that, increasing the surface fields, the growth regime changes from convex to non-defined to concave, in agreement with the interface profiles plotted in figure 3. Analogously, figures 5(a)-(d) show plots of $\cot (\theta)$ vs $T$ for $L=32$ and several different values for the magnetic field $H$. Again, vertical dashed lines correspond to transition temperatures between different growth regimes. Figure 5(a) corresponds to $H=0.2$ and displays the characteristic behavior for very small magnetic fields, that is, a convex growing interface irrespective of temperature.

For $H=0.6$ one observes a single transition from the growth regime of non- 

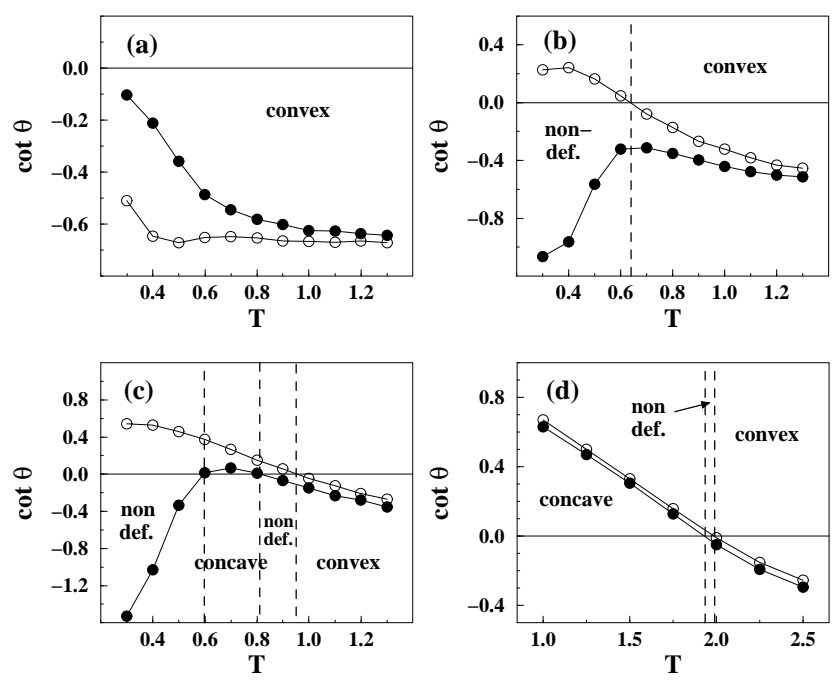

Figure 5: Plots of $\cot (\theta)$ vs $T$ for $L=32$ and several different magnetic fields: (a) $H=0.2$, (b) $H=0.6$, (c) $H=0.85$, and (d) $H=1.8 . \theta_{D}\left(\theta_{N D}\right)$ is the contact angle corresponding to the dominant (non-dominant) spin cluster, and is represented by open (filled) circles. The vertical dashed lines mark the temperatures that separate a given growth regime from another one, as indicated. Reference lines corresponding to $\cot (\theta)=0$ have also been included.

defined curvature to the convex growth regime, which shows up by increasing the temperature, as shown in figure 5(b). It should be noticed that the concave growth regime is prevented, since for small enough magnetic fields $\cot \left(\theta_{N D}\right)<0$ for all $T$. As the fields are increased, $\cot \left(\theta_{N D}\right)$ moves upwards and $\operatorname{crosses} \cot \left(\theta_{N D}\right)=0$, as expected from the plot of figure 4. For instance, the plots of $\cot (\theta)$ vs $T$ for $H=0.85$, shown in figure 5(c), exhibit this behavior. Hence, here one has to deal with three transition temperatures. Finally, by further increasing the fields, the whole lowtemperature region is dominated by the concave growth regime and two transition temperatures remain, as shown in figure $5(\mathrm{~d})$ for $H=1.8$. All these features are compactly shown in the $H-T$ phase diagram of figure 2 , where open (filled) circles refer to the transition between non-defined and convex (concave) growth regimes.

As above anticipated, we will now introduce and discuss some characteristic snapshot pictures, in order to provide qualitative explanations that account for the different growth regimes observed. Let us begin with Region $I$ (see figure 2), that corresponds to the Ising-like nonwet state and the convex growth regime. In this region, the temperature is small and the system grows in an ordered state, i.e. the dominant spin domain prevails and the deposited particles tend to have their spins all pointing in the same direction. Small clusters with the opposite orientation may appear preferably on the surface where the non-dominant orientation field is applied. 


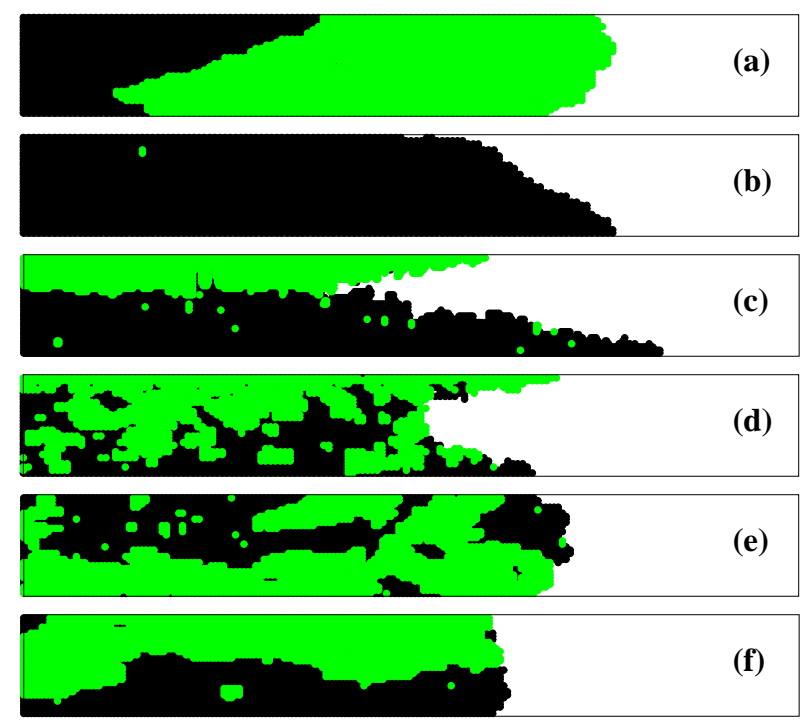

Figure 6: Typical snapshot configurations that exhibit a variety of different growth regimes. Gray (black) circles correspond to spins up (down). The surface field on the upper (lower) confinement wall is positive (negative). The snapshots correspond to a lattice size $L=32$ and several different values of temperature and surface fields: (a) $H=0.05, T=0.4$; (b) $H=0.7, T=0.4$; (c) $H=1.8, T=0.5$; (d) $H=1.8$, $T=1.0 ;(\mathrm{e}) H=0.1, T=0.7$; and (f) $H=0.3, T=0.54$.

These "drops" might grow and drive a magnetization reversal, thus changing the sign of the dominant domain (see figure 6(a)). In fact, the formation of sequences of well-ordered domains are characteristic of the ordered phase of confined (finite-size) spin systems such as the Ising magnet [4]. Due to the open boundary conditions, perimeter sites at the confinement walls experience a missing neighbor effect, that is, the number of NN sites is lower than for the case of perimeter sites on the bulk. Since $H$ is too weak to compensate this effect, the system grows preferentially along the center of the sample as compared to the walls, and the resulting growth interface exhibits a convex shape. So, Region $I$ corresponds to the Ising-like nonwet state and the convex growth regime, as shown by the snapshot picture of figure $6(\mathrm{a})$.

Let us now consider an increase in the fields, such that we enter Region $I I$ (see figure 2). Since the temperature is kept low, the system is still in its ordered phase and neighboring spins grow preferably parallel-oriented. The surface fields in this region are stronger and thus capable of compensating the missing NN sites on the surfaces. But, since the fields on both surfaces have opposite signs, it is found that, on the one hand, the field that has the same orientation as the dominant spin cluster favors the growth of surface spins, while on the other hand, the sites on the surface with opposite field have a lower probability to be chosen during the Monte Carlo growth process. Hence, the contact angle corresponding to the dominant spin cluster 
is then $\theta_{D}<\frac{\pi}{2}$, while the non-dominant is $\theta_{N D}>\frac{\pi}{2}$. Thus, on the disfavored side the growing interface becomes pinned and the curvature of the growing interface is not defined. Figure 6(b) shows a typical snapshot corresponding to Region $I I$.

Keeping $H$ fixed within Region $I I$ but increasing the temperature, thermal noise will enable the formation of drops on the disfavored side that eventually may nucleate into larger clusters as the temperature is increased even further. This process may lead to the emergence of an up-down interface, separating oppositely oriented domains, running in the direction parallel to the walls. Since sites along the up-down interface are surrounded by oppositely oriented NN spins, they have a low growing probability. So, in this case the system grows preferably along the confinement walls and the growing interface is concave (figure 6(c)). Then, as the temperature is increased, the system crosses to Region $A$ (see figure 2) and we observe the onset of two competitive growth regimes: (i) one exhibiting a non-defined growing curvature that appears when a dominant spin orientation is present, as in the case shown in figure 6(b); (ii) another that appears when an up-down interface is established and the system has a concave growth interface, as is shown in figure 6(c).

Further increasing the temperature and for large enough fields, the formation of a stable longitudinal up-down interface that pushes back the growing interface is observed. So, the system adopts the concave growth regime (see figure 6(c) corresponding to Region $I V$ in figure 2). Increasing the temperature beyond $T_{c}(L)$, a transition from a low-temperature ordered state (Region $I V$ ) to a high-temperature disordered state (Region $V I$, see figure 6(d)), both within the concave growth regime, is observed. Analogously, for small enough fields, a temperature increase drives the system from the ordered convex growth regime (Region $I$ ) to the disordered convex growth regime (Region $V$, see figure $6(\mathrm{e})$ ). As shown in figure 2 , there is also an intermediate fluctuating state (Region $B$ ) between Regions $V$ and $V I$, characterized by the competition between the disordered convex growth regime and the disordered concave one.

Finally, a quite unstable and small region (Region $I I I$ in figure 2) that exhibits the interplay among the growth regimes of the contiguous regions, can also be identified. Since the width of Region $I I I$ is of the order of the rounding observed in $T_{c}(L)$, large fluctuations between ordered and disordered states are observed, as well as from growth regimes of non-defined curvature to convex ones. However, figure $6(\mathrm{f})$ shows a snapshot configuration that is the fingerprint of Region $I I I$, namely a well defined spin up-down interface with an almost flat growing interface.

As already commented, the noncriticality of confined $(1+1)$ - dimensional magnetic Eden films implies that the whole ordered phase corresponding to the $T<T_{c}(L)$ region, which contains the quasi-wetting curve, collapses in the $L \rightarrow \infty$ limit, since in this limit $T_{c}(L) \rightarrow 0$ [35]. Concerning the structure of the disordered phase in the thermodynamic limit, it can be shown that the morphological transition curves merge into a single curve that separates the disordered convex 


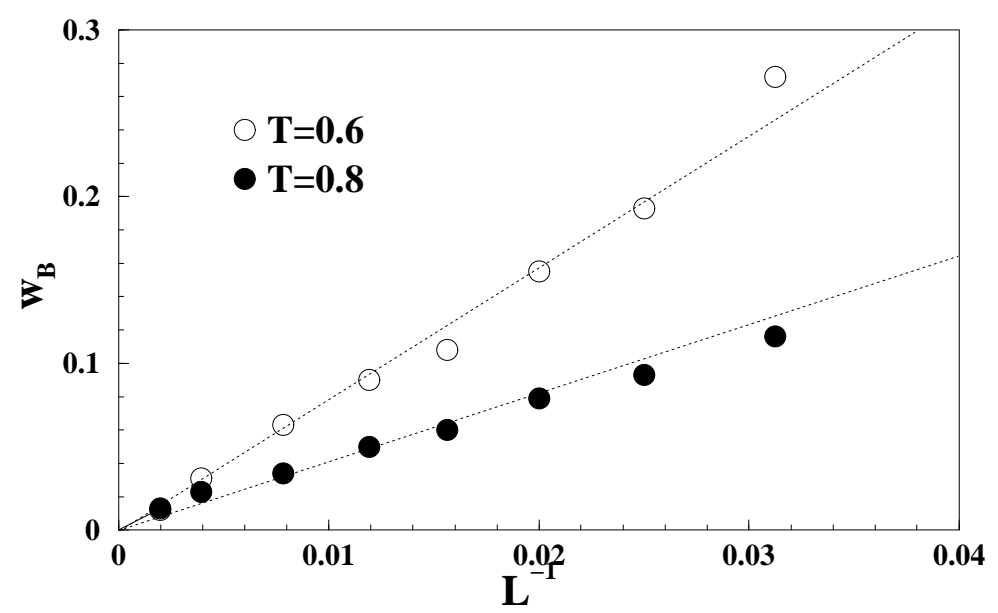

Figure 7: Plots of the isothermal width of Region $B\left(w_{B}(L, T)\right)$ versus $L^{-1}$ for lattice sizes in the range $32 \leq L \leq 512$, corresponding to $T=0.6$ and $T=0.8$. The lines are guides to the eye. Following this extrapolation procedure, it turns out that Region $B$ collapses in the $L \rightarrow \infty$ limit, and so only Regions $V$ and $V I$ are present in the infinite size phase diagram.

growth regime (Region $V$ ) from the disordered concave one (Region $V I$ ). In order to illustrate this, we define $w_{B}(L, T)$ as the isothermal width of the intermediate fluctuating state (Region $B$ ). For instance, $w_{B}$ is marked in figure 2 for $L=32$ and $T=0.6$. Figure 7 shows plots of $w_{B}(L, T)$ versus $L^{-1}$ corresponding to lattice sizes in the range $32 \leq L \leq 512$ and for two different temperatures. Using this standard extrapolation procedure, it turns out that $w_{B}(L, T) \rightarrow 0$ as $L \rightarrow \infty$, and so only Regions $V$ and $V I$ are present in the infinite size phase diagram.

\section{Conclusions}

The growth of magnetic Eden thin films with ferromagnetic interactions between nearest-neighbor spins has been studied in a $(1+1)$-dimensional geometry with competing surface magnetic fields. Besides an Ising-like quasi-wetting transition, two morphological transitions in the growing interface, which arise from the MEM's kinetic growth process, have also been identified. The resulting phase diagram exhibits eight regions. Among them, six correspond to well defined growth regimes, which are illustrated by typical snapshot pictures. The remaining two regions appear as fluctuating crossover states characterized by the competition between the growth regimes of neighboring regions. We hence conclude that the nonequilibrium nature of growing magnetic Eden thin films introduces new and rich physical features of interest, as compared to wetting phase diagrams for equilibrium spin systems (e.g. 
the well-studied Ising model [4]). In the thermodynamic limit, the whole ordered phase (which contains the quasi-wetting curve) collapses. Indeed only two regions, which correspond to the disordered convex growth regime and the disordered concave one, are present in the phase diagram of the infinite system.

We expect that the present study will contribute to the fields of nonequilibrium wetting phenomena and irreversible growth processes in confined geometries, and we hope that it will stimulate further experimental and theoretical work in these topics of widespread technological and scientific interest.

\section{Acknowledgments}

This work was supported by CONICET, UNLP, and ANPCyT (Argentina).

\section{References}

[1] J. Shen et al., Phys. Rev. B 56, 2340 (1997).

[2] J.-S. Tsay and Y.-D. Yao, Appl. Phys. Lett. 74, 1311 (1999).

[3] O. Pietzsch, A. Kubetzka, M. Bode and R. Wiesendanger, Phys. Rev. Lett. 84, $5212(2000)$.

[4] E.V. Albano, K. Binder, D. Heermann, and W. Paul, Surf. Sci. 223, 151 (1989).

[5] D. Karevski and M. Henkel, Phys. Rev. B 55, 6429 (1997).

[6] J.T. Ou, F. Wangand, and D.L. Lin, Phys. Rev. E 56, 2805 (1997).

[7] F.D.A. Aarão Reis, Phys. Rev. B 58, 394 (1998) and 626565 (2000).

[8] U. Steiner, E. Eiser, J. Klein, A. Budkowski, and L.J. Fetters, Science 258, 1126 (1992).

[9] U. Steiner and J. Klein, Phys. Rev. Lett. 77, 2526 (1996).

[10] A.F.G. Wyatt, J. Klier, and P. Stefanyi, Phys. Rev. Lett. 74, 1151 (1995).

[11] P.G. de Gennes, Rev. Mod. Phys. 57, 827 (1985).

[12] S. Dietrich, in "Phase Transitions and Critical Phenomena", C. Domb and J.L. Lebowitz (Eds.), Academic Press, New York, Vol.12 (1988). 
[13] G. Forgacs, R. Lipowsky, and Th.M. Nieuwenhuizen, in "Phase Transitions and Critical Phenomena", C. Domb and J.L. Lebowitz (Eds.), Academic Press, London, Vol.14 (1991).

[14] A.O. Parry, J. Phys. Cond. Matter 8, 10761 (1996).

[15] A.O. Parry and R. Evans, Phys. Rev. Lett. 64, 439 (1990); Physica A 181, 250 (1992).

[16] M.R. Swift, A.L. Owczarek, and J.O. Indekeu, Europhys. Lett. 14, 465 (1991).

[17] A. Maciolek and J. Stecki, Phys. Rev. B 54, 1128 (1996).

[18] A. Maciolek, J. Phys. A (Math. and Gen.) 29, 3837 (1996).

[19] E. Carlon and A. Drzewiński, Phys. Rev. E 57, 2626 (1998).

[20] H.L. Frisch, S. Puri, and P. Niebala, J. Chem. Phys. 110, 10514 (1999).

[21] H. Liu, A. Bhattacharya, and A. Chakrabarti, J. Chem. Phys. 109, 8607 (1998).

[22] M. Mueller, E.V. Albano, and K. Binder, Phys. Rev. E 62, 5281 (2000).

[23] K. Binder, D.P. Landau, and A.M. Ferrenberg, Phys. Rev. Lett. 74, 298 (1995); Phys. Rev. E 51, 2823 (1995).

[24] K. Binder, D.P. Landau, and A.M. Ferrenberg, Phys. Rev. E 53, 5023 (1995).

[25] A. Wener, F. Schmid, M. Mueller, and K. Binder, J. Chem. Phys. 107, 8175 (1997).

[26] A.M. Ferrenberg, D.P. Landau, and K. Binder, Phys. Rev. E 58, 3353 (1998).

[27] U. Bovensiepen et al., Phys. Rev. Lett. 81, 2368 (1998).

[28] Notice that $M$ is the growing direction of the magnetic film that plays the role of the growing "time". After reaching the stationary growing regime this variable is irrelevant for the computation of averaged quantities and consequently does not enter in the scaling functions.

[29] M. Eden, in "Symp. on Information Theory in Biology", H.P. Yockey (Ed.), Pergamon Press, New York (1958); "Proceedings of the Fourth Berkeley Symposium on Mathematics, Statistics and Probability", F. Neyman (Ed.), University of California Press, Berkeley, Vol.IV, p.223 (1961).

[30] Fractals and Disordered Media, A. Bunde and S. Havlin (Eds.), Springer-Verlag, Heidelberg (1991). 
[31] Fractals in Science, A. Bunde and S. Havlin (Eds.), Springer-Verlag, Heidelberg (1995).

[32] A.L. Barabasi and H.E. Stanley, Fractal Concepts in Surface Growth, Cambridge University Press, New York (1995).

[33] M. Marsili, A. Maritan, F. Toigo, and J.R. Banavar, Rev. Mod. Phys. 68, 963 (1996).

[34] M. Ausloos, N. Vandewalle, and R. Cloots, Europhys. Lett. 24, 629 (1993).

[35] J. Candia and E.V. Albano, Phys. Rev. E 63, 066127 (2001). 